OPEN ACCESS

Edited by: Leonidas Stamatatos, Fred Hutchinson Cancer Research

Center, United States

Reviewed by:

Tomasz Brudek, Bispebjerg Hospital, Denmark Shivashankar Othy, University of California, Irvine, United States

*Correspondence: Lifen Yao ylf0431@163.com Huan Nie nh1212@hit.edu.cn

Qinghua Jiang qhjiang@hit.edu.cn

Specialty section: This article was submitted to Systems Immunology, a section of the journal

Frontiers in Immunology

Received: 13 November 2021 Accepted: 27 January 2022

Published: 16 February 2022

Citation:

Wang $P$, Luo $M$, Zhou $W$, Jin $X, X u Z$, Yan S, Li Y, Xu C, Cheng R, Huang Y, Lin X, Yao L, Nie H and Jiang Q (2022) Global Characterization of Peripheral $B$ Cells in Parkinson's Disease by SingleCell RNA and BCR Sequencing. Front. Immunol. 13:814239. doi: 10.3389/fimmu.2022.814239

\section{Global Characterization of Peripheral B Cells in Parkinson's Disease by Single-Cell RNA and BCR Sequencing}

Pingping Wang ${ }^{1}$, Meng Luo ${ }^{1}$, Wenyang Zhou ${ }^{1}$, Xiyun Jin ${ }^{1}$, Zhaochun $X u^{1}$, Shi Yan ${ }^{2}$, Yiqun $\mathrm{Li}^{1}$, Chang $\mathrm{Xu}^{1}{ }^{,}$, Rui Cheng ${ }^{1}$, Yan Huang ${ }^{1}$, Xiaoyu Lin $^{1}$, Lifen Yao ${ }^{2 *}$, Huan Nie ${ }^{1 *}$ and Qinghua Jiang ${ }^{1,3 *}$

\footnotetext{
${ }^{1}$ School of Life Science and Technology, Harbin Institute of Technology, Harbin, China, ${ }^{2}$ Department of Neurology, First Affiliated Hospital of Harbin Medical University, Harbin, China, ${ }^{3}$ Key Laboratory of Biological Big Data (Harbin Institute of Technology), Ministry of Education, Harbin, China
}

Immune system plays important roles in the pathogenesis of Parkinson's disease (PD). However, the role of B cells in this complex disease are still not fully understood. B cells produce antibodies but can also regulate immune responses. In order to decode the relative contribution of peripheral $B$ cell subtypes to the etiology of $P D$, we performed single cell RNA and BCR sequencing for 10,466 B cells from 8 PD patients and 6 agematched healthy controls. We observed significant increased memory B cells and significant decreased naïve B cells in PD patients compared to healthy controls. Notably, we also discovered increased $\lg G$ and $\lg A$ isotypes and more frequent class switch recombination events in PD patients. Moreover, we identified preferential $\mathrm{V}$ and $\mathrm{J}$ gene segments of $\mathrm{B}$ cell receptors in $\mathrm{PD}$ patients as the evidence of convergent selection in $\mathrm{PD}$. Finally, we found a marked clonal expanded memory $B$ cell population in $P D$ patients, up-regulating both MHC II genes (HLA-DRB5, HLA-DQA2 and HLA-DPB1) and transcription factor activator protein 1 (AP-1), suggesting that the antigen presentation capacity of B cells was enhanced and B cells were activated in PD patients. Overall, this study conducted a comprehensive analysis of peripheral B cell characteristics of PD patients, which provided novel insights into the humoral immune response in the pathogenesis of PD.

Keywords: Parkinson's disease, B cells, scRNA-seq, scBCR-seq, adaptive immune response

\section{INTRODUCTION}

Parkinson's disease (PD) is a progressive central nervous system disorder that affects the movement (1). The main motor symptoms are rigidity, tremor, slow movement, and difficulty in walking (1). Mental and behavioral changes may also accompanied with sleep problems, depression, memory difficulties, and fatigue (1). It is estimated that $1 \%$ of people over the age of 60 suffer from $\operatorname{PD}(2,3)$. About 5 to 10 percent of patients are diagnosed before the age of $50(4,5)$. Overall, about 10 million 
people around the world currently suffer from PD (6), and up to 80 percent of PD patients will eventually develop dementia (7).

The pathological hallmarks of PD are $\alpha$-synuclein aggregation and Lewy body formation, resulting in the gradual loss of dopaminergic neurons in the substantia nigra (8). Increasing studies have shown that immune system dysfunction plays a critical role in PD pathophysiology (9). Specific variants in the HLA region are associated with PD (10, 11 ), and $\alpha$-synuclein specific T cells were found to be involved in the pathogenesis of PD $(12,13)$. The levels of activated T cells are increased both in the blood and cerebrospinal fluid (CSF) of PD patients $(14,15)$, and $\mathrm{T}$ cells can also be detected in the midbrains of PD patients (16). The potential role of $\mathrm{B}$ cells in PD is also emerging (17). Chronic and acute MPTP administration alleviated DA neuronal loss and behavioral disorders in RAG2 knockout mice lacking both T and B cells $(16,18)$. IgG deposits on dopaminergic neurons has been observed in PD patients, and Lewy bodies were also coated with $\operatorname{IgG}$, indicating that dopaminergic neurons might be the targets of these immunoglobulins (19). In addition, elevated levels of anti- $\alpha$-synuclein antibodies can also be detected in the blood and cerebrospinal fluid of PD patients $(20,21)$. MPTPtreated mice produced natural and nitrated $\alpha$-synuclein antibodies (22). IgG obtained from PD patients caused selective dopaminergic neuron loss (23). Although these evidences indicate that humoral immunity plays a potential role in $\mathrm{PD}$, the relative contribution of peripheral $\mathrm{B}$ cell subtypes to the etiology of PD is still unclear.

$\mathrm{B}$ cells produce antibodies but can also regulate immune responses. Since infiltrating B cells have not been detected in the brains of PD patients (16), B cells may participate in central inflammation through their activities in the periphery. In this study, we conducted single-cell RNA and BCR sequencing to systematically characterize the cellular composition, immunoglobulin isotypes, preferential $\mathrm{V}$ and $\mathrm{J}$ gene segments and clonal expansion of peripheral B lymphocytes in PD patients. This large-scale single-cell expression and immune profiling data of B cells can be used as valuable resources to study the basic humoral immune response in the disease pathogenesis and potentially guide the effective diagnosis and immunotherapy strategies for PD.

\section{RESULTS}

\section{Single-Cell RNA and BCR Profiling of B Cells in Parkinson's Disease}

We comprehensively analyzed the single-cell RNA and BCR profiling of $\mathrm{B}$ cells in the blood of patients with $\mathrm{PD}$ and healthy controls (Figure 1A). Detailed information of PD patients was described in Supplementary Table S1. Peripheral blood mononuclear cells (PBMCs) were isolated from fresh blood of $8 \mathrm{PD}$ patients and 6 healthy controls. CD19+ B lymphocytes were sorted by flow cytometry, and single-cell 5' gene-expression libraries and $\mathrm{V}(\mathrm{D}) \mathrm{J}$ enriched libraries were prepared using a 10x Genomics single-cell immune profiling workflow.
After removing low-quality cells, we finally obtained single-cell expression data for 10,466 B cells, comprising 6,681 cells (mean: 835 cells) for PD patients and 3,785 cells (mean: 631 cells) for healthy controls (Supplementary Table S1). In addition, we obtained 13,957 single-cell paired BCRs, comprising 8,704 BCRs (mean: 1088 BCRs) from PD patients and 5,253 BCRs (mean: 876 BCRs) from healthy controls (Supplementary Table S1). 10,206 B cells have paired gene expression and BCR profiles (Supplementary Table S1).

\section{Altered B Cell Composition and Transcriptome in Parkinson's Disease}

In order to reveal the internal cellular composition and functional status of peripheral blood B cells, different B cell populations were identified using an unsupervised clustering approach embedded in Seurat $(24,25)$. B cells were visualized in t-distributed stochastic neighbor embedding ( $\mathrm{t}-\mathrm{SNE}$ ) based on the gene expression profiling. In total, we identified 9 distinct clusters representing different B cell subtypes (Figure 1B). B cells were annotated by manually checking the cell-identity marker expression and their global similarity with the gene expression of reference datasets (2630) by SingleR (31). Spearman correlation between single cell and bulk RNA-seq expression profiles were computed, and the cell labels were transferred from bulk RNA-seq datasets to every single cell. Each dataset was used separately to calculate the correlation. In most cases, a cluster was annotated by the labels of majority of cells from that cluster (Supplementary Figure S1). We identified the major cell types of B cells, including: naïve B cells (IgD+CD27-) (Naïve1, Naïve2, Naïve3, Naïve4 clusters), unswitched memory B cells (IgD+CD27+) (USM1, USM2 clusters), switched memory B cells (IgD-CD27+) (SM1, SM2 clusters) and plasmablast/plasma cells (IgD-CD27hi) (PC cluster) (Figures 1B, C). For unswitched memory B cells, compared to USM1 subset, USM2 overexpressed several marker genes related to B cell activation, proliferation, and differentiation, such as FCRL5 (32), EGR1 (33) and CD86 (34) (Supplementary Figure S2A). For switched memory B cells, SM1 and SM2 had distinct gene expression patterns (Supplementary Figure S2B). SM1 overexpressed CD24, which induces apoptosis in human B cells through interactions with glycolipid-enriched membrane domains (35), while SM2 highly expressed CD99, an activation-associated molecule that is upregulated in recently activated lymphocytes (36) (Supplementary Figure S2B). Overall, the USM2 and SM2 subsets of memory cells appear to be activated $\mathrm{B}$ cell populations, and we will focus on their composition and functional status in patients with Parkinson's disease. To further understand the relationships among $\mathrm{B}$ cell subsets, we used Monocle (37) to perform pseudo-time ordering for these cells and visualize them on the first two principal components (Figure 1D). A process of transformation from naïve B cells to unswitched and switched memory B cells followed by plasma cells is clearly observed (Figure 1D). And this lineage directionality was further confirmed by RNA velocities (Figure 1D).

In order to understand the composition of $\mathrm{B}$ cell populations in $\mathrm{PD}$ patients, we compared the percentage of $\mathrm{B}$ cell subsets in $\mathrm{PD}$ patients and healthy controls. B cells exhibit a specific composition in PD patients (Figure 1E). We observed 
significant increase of unswitched memory B cells (Wilcox test, two-sided, $p=0.0027$ ) and significant decrease of naïve B cells (Wilcox test, two-sided, $p=0.0047$ ) in PD patients compared to healthy controls (Figure 1F). In PD patients, naïve B cells accounted for $39 \%$ of the total B cells, while in healthy controls, this proportion rose to 64\% (Figure 1G). While, unswitched memory and switched memory B cells accounted for $60.1 \%$ of total B cells in PD patients, which was nearly twice of that in healthy controls (Figure 1G).

\section{Immunoglobulin Isotypes and Class- Switching Events of BCRs in PD Patients}

To explore B cell immunoglobulin (Ig) repertoire, we investigated Ig heavy-chain isotypes for PD patients and healthy controls. Four main types of Ig were detected from scBCR-seq data, including IgD, IgM, IgA and IgG. IgM had the largest proportion in naïve $\mathrm{B}$ cells and unswitched memory $\mathrm{B}$ cells, while IgG and IgA were mainly distributed in switched memory B cells and plasma cells (Figure 2A). In PD patients,
IgM accounted for $57 \%$ of the total BCR isotypes, while in healthy controls, this proportion rose to 67\% (Figure 2B). In addition, IgG accounted for $22 \%$ of total BCR isotypes in PD patients, which was 1.8 folds of that in healthy controls (Figure 2B). The ratios of $\operatorname{IgG}$ to $\operatorname{IgM} / \mathrm{D}$ and $\operatorname{IgA}$ to $\operatorname{IgM} / \mathrm{D}$ in $\mathrm{PD}$ patients were almost twice of those in healthy controls (Figure 2C). Chi-squared test further confirmed the significant association between disease and different BCR isotypes (Pearson's chi-squared test, X-squared $=244.06, \mathrm{df}=8$, simulated $\mathrm{p}<2.2 \mathrm{E}-16$, Figure 2D). The isotypes IgG1 and IgG3 were significantly associated with PD patients, while IgM and $\operatorname{IgD}$ was significantly associated with healthy controls (Figure 2D). Since antibodies that undergo Ig class switch recombination (CSR) and somatic hypermutation (SHM) have higher affinity and longer-lasting protection (38), we assigned BCRs to clonotypes and performed comparison analysis of CSR events in each clonotype between PD patients and healthy controls. Clonotypes were defined by clustering Ig sequences using the DefineClones function in Change-O toolkit (39). We

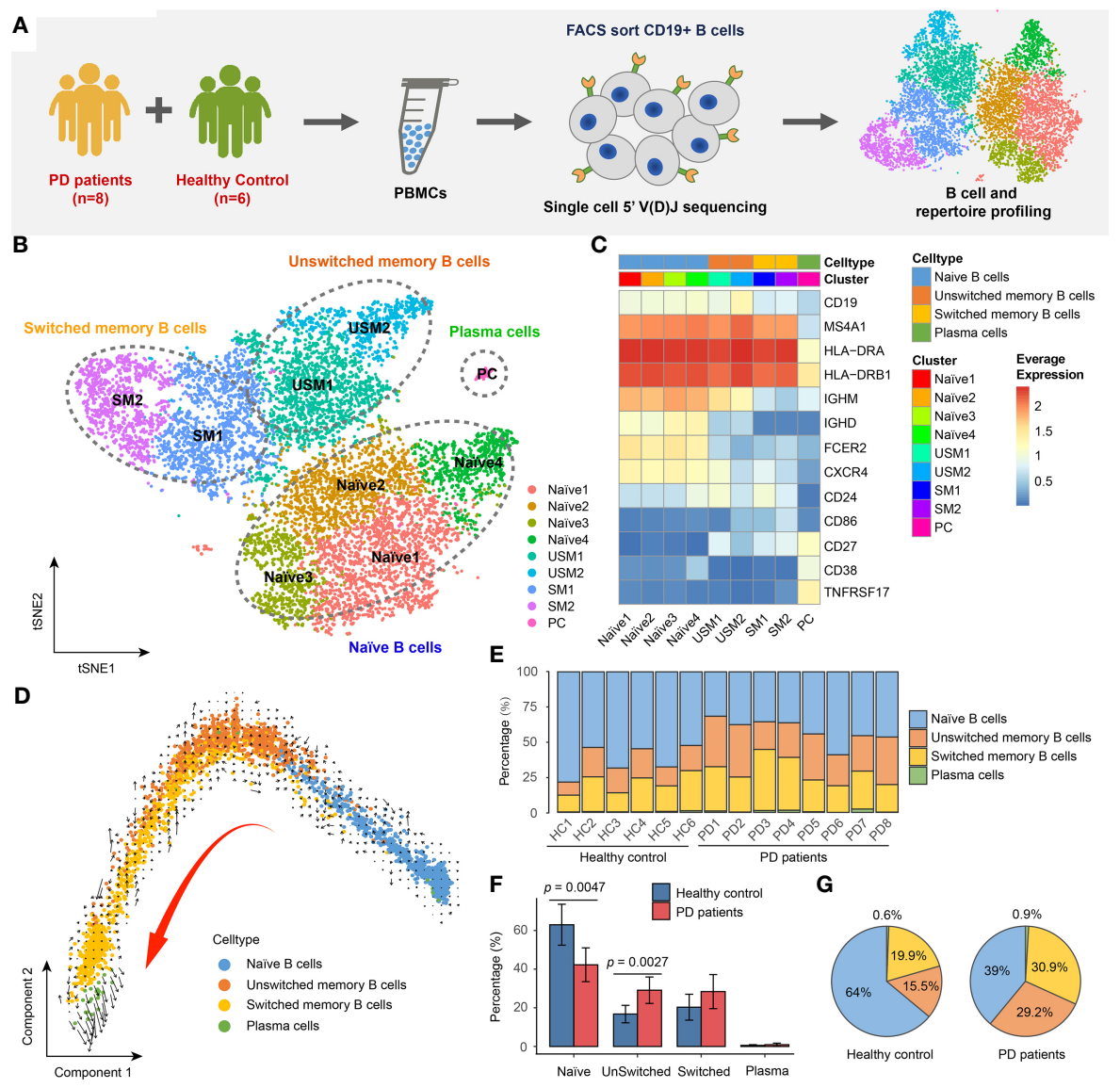

FIGURE 1 | Landscape of Peripheral blood B cells in Parkinson's disease revealed by single-cell transcriptome sequencing. (A) Overview of experimental design. CD19+ B cells were sorted by FACS and then subjected to single-cell RNA and V(D)J sequencing. (B) tSNE projection of 10,466 single B cells, showing 9 distinct clusters. (C) Heatmap shows the average logCPM of classical marker genes for all 9 cell clusters. (D) The velocities are visualized on the first two principal components calculated by monocle. (E) Bar chart shows the percentages of four major types of B cell in each sample. (F) Bar plot shows the average percentages of four major B cell types in PD patients and healthy controls. Error bars represent the standard deviation. (G) Pie charts show the percentage composition of B cells in PD patients and healthy controls. 


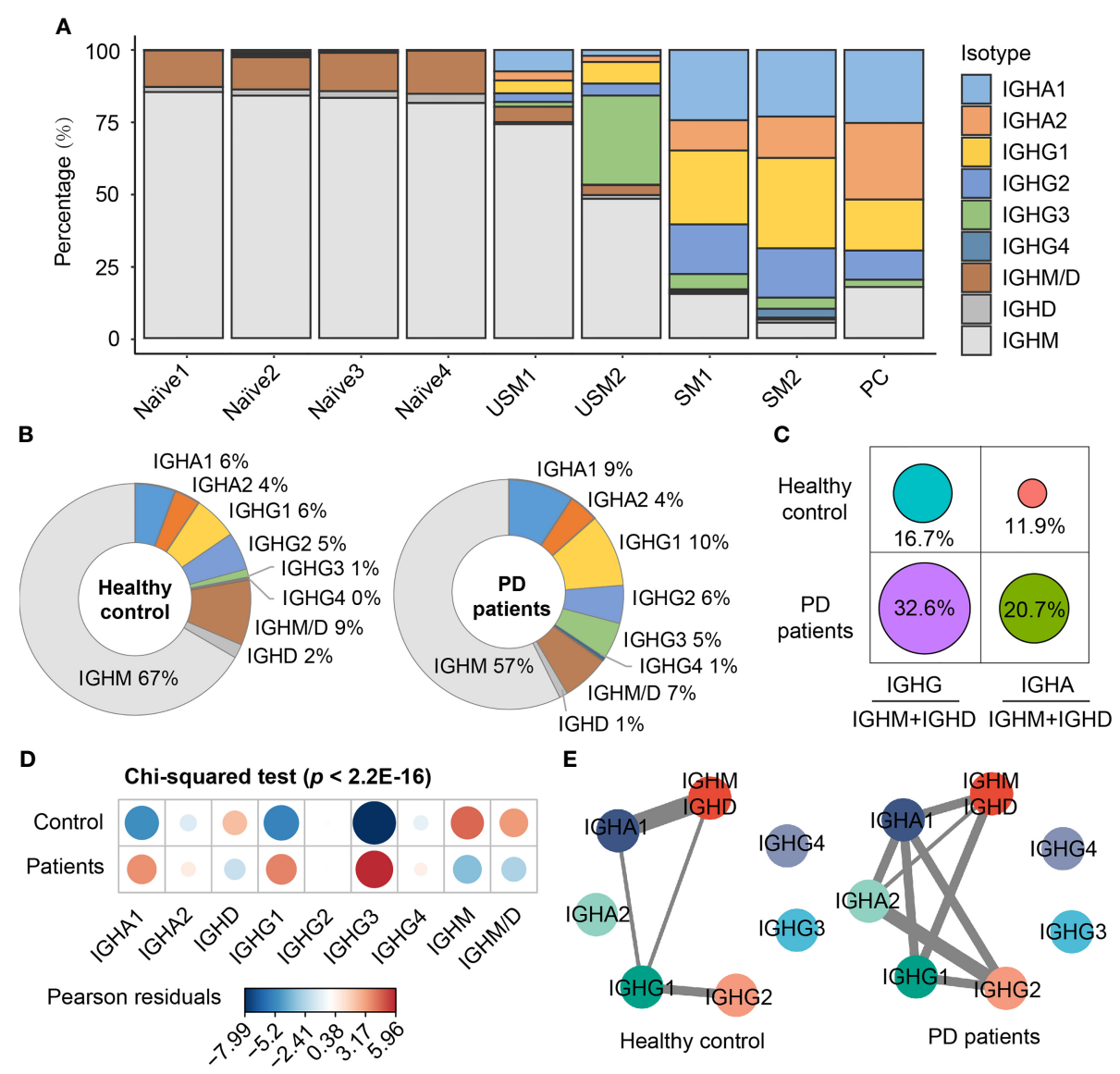

FIGURE 2 | Immunoglobulin isotypes and class-switching events of BCRs in PD patients and healthy controls. (A) Immunoglobulin isotype distribution in each B cell subsets. (B) Pie chart shows the percentage composition of Ig isotypes in PD patients and healthy controls. (C) The ratio of $\lg$ to $\lg M / D$ and $\lg A$ to $\lg M / D$ between PD patients and healthy controls. (D) Bubble chart shows the Pearson's residuals of sample groups from Ig isotypes. Red circles indicate an over-representation, and blue circles indicate an under-representation. (E) Class-switching events in PD patients and healthy controls. The thickness of the line indicates the number of sharing clonotypes between two lg isotypes.

observed more CSR events in PD patients, especially IgM to IgG and IgA to IgG (Figure 2E). These changes suggest an activated humoral immune response in the peripheral blood of PD patients.

\section{Preferential V and J Gene Segments of BCRs in PD Patients}

In order to search for the evidence of convergent antibody evolution in $\mathrm{PD}$, we compared the preferential gene segment usage in $\mathrm{V}(\mathrm{D}) \mathrm{J}$ rearrangements between PD patients and healthy controls. B cells undergo $\mathrm{V}(\mathrm{D}) \mathrm{J}$ recombination of variable $(\mathrm{V})$ and joining $(J)$ gene segments in the light $(\mathrm{L})$ chain $(\kappa$ and $\lambda$ ), and of variable $(\mathrm{V})$, diversity $(\mathrm{D})$, and joining (J) gene segments in the heavy $(\mathrm{H})$ chain in order to generate diverse repertoires of $\mathrm{B}$ cell receptors capable of recognizing a wide range of pathogen epitopes (40).

For $\mathrm{V}_{\mathrm{H}}$ gene segments, the frequencies of $18 \mathrm{~V}_{\mathrm{H}}$ gene segments were significantly different between PD patients and healthy controls, of which, $7 \mathrm{~V}_{\mathrm{H}}$ gene segments (IGHV2-5, IGHV1-3, IGHV4-61, etc.) increased in PD patients, while 11
$\mathrm{V}_{\mathrm{H}}$ gene segments (IGHV4-59, IGHV3-34, IGHV1-18, etc.) increased in healthy controls (Figure 3A). For $V_{L}$ gene segments, $10 \mathrm{~V}_{\mathrm{L}}$ gene segments were significantly different between PD patients and healthy controls, of which $5 \mathrm{~V}_{\mathrm{L}}$ gene segments (IGLV3-1, IGKV6-21, IGLV2-8, etc.) increased in PD patients and $5 \mathrm{~V}_{\mathrm{L}}$ gene segments (IGLV1-51, IGLV1-44, etc.) increased in healthy controls (Figure 3B). For $\mathrm{J}_{\mathrm{H}}$ gene segments, only IGHJ3 was significantly increased in PD patients (Figure 3C). For $\mathrm{J}_{\mathrm{L}}$ gene segments, IGKJ2 and IGLJ3 were significantly increased in PD patients, while IGLJ2 and IGKJ3 were significantly increased in healthy controls (Figure 3D).

Then, we further compared the VJ pairing of heavy and light chains separately. For heavy chain, $6 \mathrm{VJ}_{\mathrm{H}}$ pairs (IGHV6-1/ IGHJ6, IGHV4-34/IGHJ3, etc.) were significantly increased in PD patients, while $9 \mathrm{VJ}_{\mathrm{H}}$ pairs (IGHV1-18/IGHJ5, IGHV3-11/ IGHJ3, etc.) were significantly increased in healthy controls (Figures 3E, G). For light chain, $3 \mathrm{VJ}_{\mathrm{L}}$ pairs (IGLV2-11/IGLJ3, IGKV3D-15/IGKJ4 and IGKV3D-20/IGKJ1) were significantly increased in PD patients, while $6 \mathrm{VJ}_{\mathrm{L}}$ pairs (IGKV1D-13/IGKJ2, IGKV3-15/IGKJ5, etc.) were significantly increased in healthy 
A

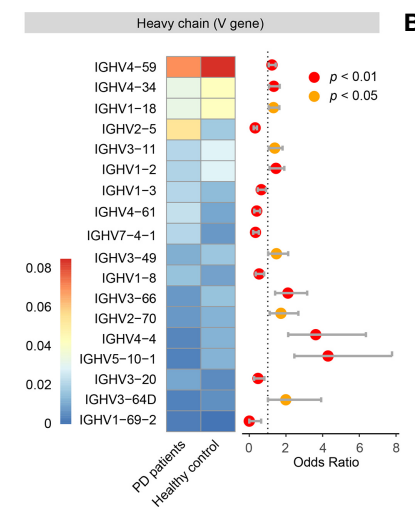

E

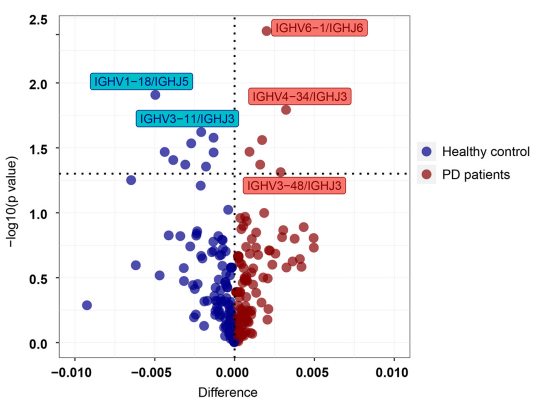

B
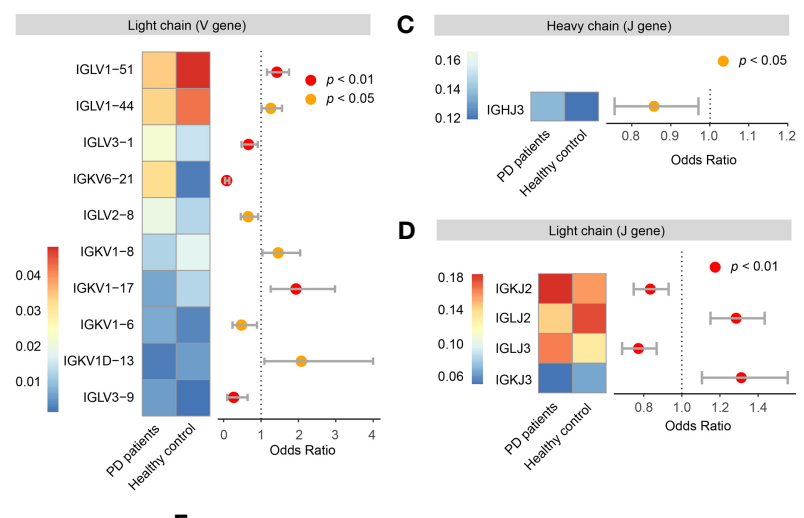

D
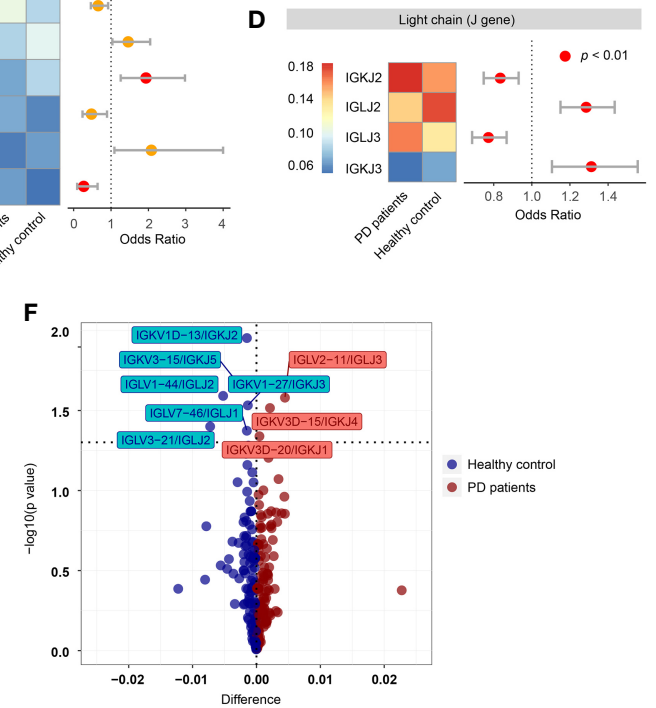

G
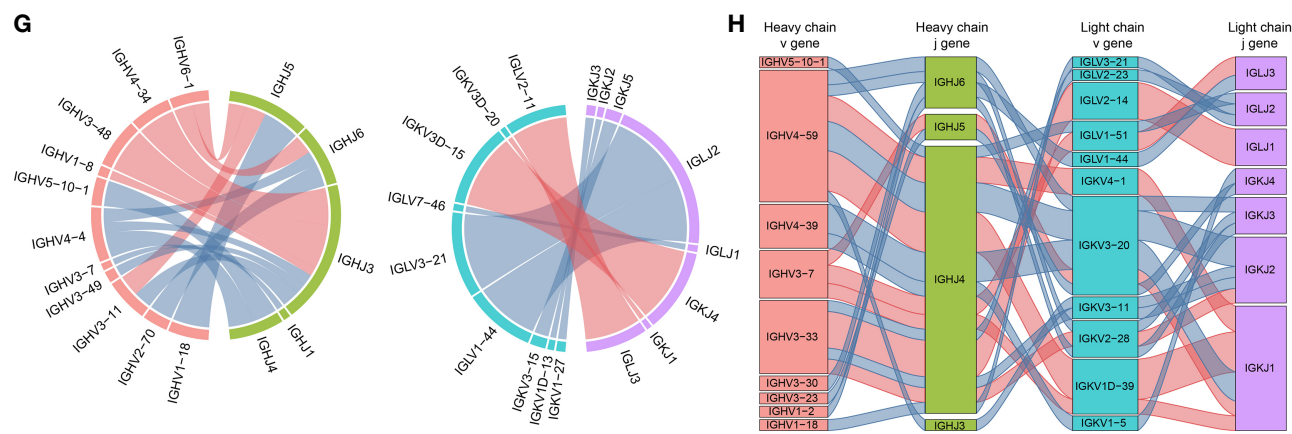

FIGURE 3 | Preferential variable ( ) and joining (J) gene segments in the heavy and light chain of BCRs in PD patients. (A) Differential usage (Fisher's exact test) of $V_{H}$ gene segments between PD patients and healthy controls. (B) Similar to A, differential usage of $V_{L}$ gene segments. (C) Similar to A, differential usage of $J_{H}$ gene segments. (D) Similar to A, differential usage of $J_{L}$ gene segments. (E) Volcano plot shows the significant different frequency of heavy chain $V J$ pairs of PD patients compared to healthy controls. (F) Similar to E, volcano plot shows the significant different frequency of light chain $\mathrm{VJ}$ pairs. (G) Circos plots show the differential heavy (left) and light (right) VJ pairs in PD patients and healthy controls. Red links represent PD specific VJ pairs, and blue links represent healthy control specific VJ pairs. (H) Sankey diagram shows significant different frequency of heavy-light VJ pairs in PD patients and healthy controls. Red links represent PD specific pairs, and blue links represent healthy control specific pairs.

controls (Figures 3F, G). Of all the heavy-light VJ pairs, 20 heavy-light VJ pairs were significantly different between PD patients and healthy controls, of which 7 heavy-light VJ pairs increased in PD patients, others decreased (Figure 3H).

\section{A Marked Clonal Expansion of Memory B Cells in PD Patients}

In order to better understand the activated $\mathrm{B}$ cell types in $\mathrm{PD}$, we conducted a comparison analysis of the $\mathrm{B}$ cell clonal expansion between PD patients and healthy controls. In total, we detected 13,957 BCRs, forming 12,938 unique clonotypes, of which 357 clonotypes detected in at least two cells, indicating clonal expansion of peripheral blood B cells. $7.2 \%(5.4 \%+1.8 \%)$ of B cells were clonally expanded in PD patients, which was higher than $5.2 \%(3.8 \%+1.4 \%)$ in healthy controls (Figure $\mathbf{4 A})$. The UMAP plot shows that clonal expansion mainly occurs in memory B cells, especially the unswitched memory B cell cluster USM2 (Figure 4B). Fisher's exact test further confirmed the significance of the clonal expansion of unswitched memory B cell cluster USM2 (Fisher's exact test, two-sided, FDR = 1.29E-12) (Figures 4B, C). In PD patients, B cell cluster USM2 tend to have much larger clonotypes with 416 clonotypes detected from $626 \mathrm{~B}$ cells (an average of $1.5 \mathrm{~B}$ cells per clonotype), while in healthy controls the average clone size was 1 (106 clonotypes out of 109 B cells) (Figure 4D). Antibodies produced by unswitched and switched memory B cells from the 
same clonotypes retain affinity for the same antigens (41). Since antibody class switching diversifies the effector properties of antibodies (41), clonal expansion of memory B cells (especially unswitched memory B cells) in PD patients may be a strategy to cope with the increasing central nervous system inflammation.

To investigate the function of $B$ cell populations in $\mathrm{PD}$, we conducted differentially expressed analysis for each B cell cluster between PD patients and healthy controls (Supplementary Table S2). Gene Ontology and KEGG pathway enrichment analyses of the differentially expressed genes (DEGs) were performed (Supplementary Table S2). DEGs enriched in B cell receptor signaling pathway (hsa04662), antigen processing and presentation (hsa04612) and cell adhesion molecules (hsa04514) were selected to further view their expression profiles in each cluster (Figure 4E). We observed that MHC II genes (HLA-DRB5, HLA-DQA2 and HLA-DPB1) were significantly overexpressed in $\mathrm{B}$ cells of $\mathrm{PD}$ patients, especially in memory B cells and plasma cells, indicating their antigen presentation function were enhanced in PD patients (Figure 4E). It is generally believed that B cells as APCs are less effective than dendritic cells and other myeloid cells, but when the antigen recognized by BCR is presented to $\mathrm{T}$ cells that recognize the same antigen, the antigen presentation efficiency of memory B cells increases (42-44). In addition, memory B cells and plasma cells in PD patients also up-
A

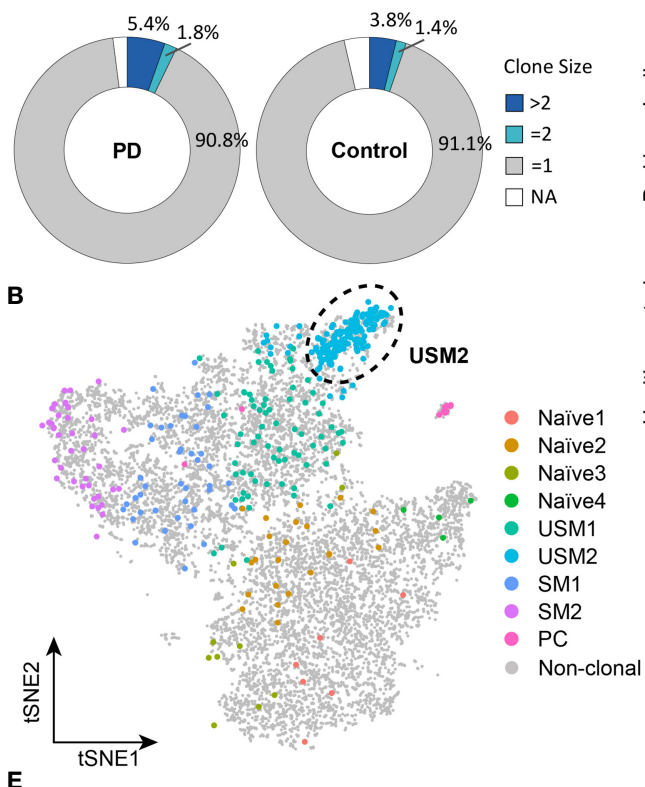

E

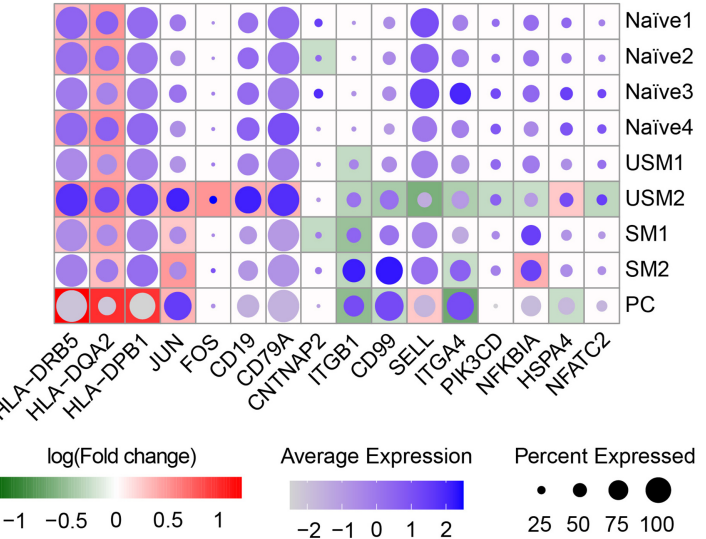

c
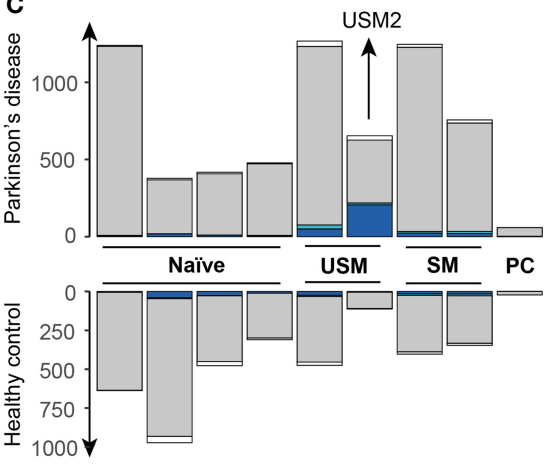

D
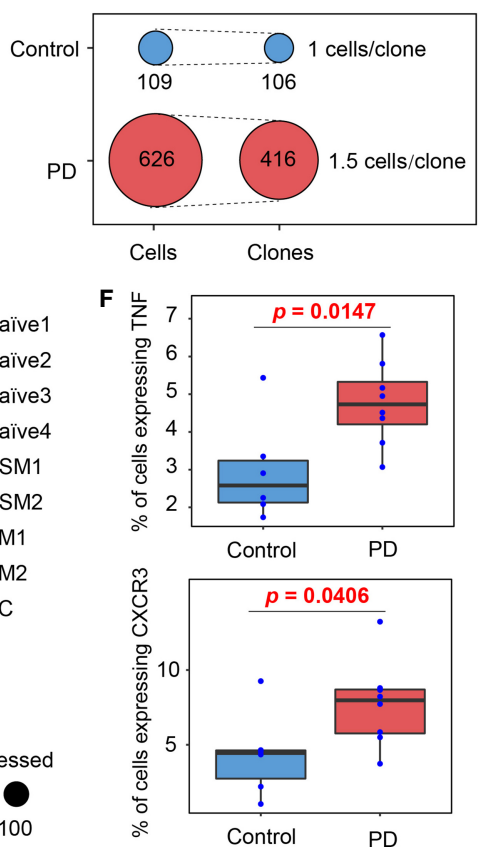

FIGURE 4 | Clone expansion of B cells in Parkinson's disease. (A) Pie charts show the distribution of clonotypes grouped by clone size (NA, = 1, = 2, $>2, \mathrm{NA}$ represents cells with no BCR sequence detected). (B) tSNE plot shows the distribution of clonally expanded B cells. (C) Bar plots show the distribution of clonotypes grouped by clone size in PD patients and healthy controls. (D) Clonotype diversity of unswitched memory B cells (USM2) in PD patients and healthy controls. (E) A global view of the differentially expressed genes related to B cell receptor signaling pathway in each cluster. The size of the dot represents the percentage of cells expressing the gene in each cluster, while the color represents the average gene expression value. Background heatmap shows the logtransformed fold-change of DEGs between PD patients and healthy controls. (F) Box plots show the percentages of B cells expressing TNF and CXCR3 in PD patients and healthy controls. Each dot represents a sample. 
regulated activator protein 1 (AP-1) transcription factors (JUN and FOS) (Figure 4E), which controls a number of cellular processes including differentiation, proliferation, and apoptosis during B-cell activation (45). These results suggest that the antigen presentation capacity of B cells was enhanced and B cells were activated in PD patients.

\section{DISCUSSION}

Recently, neuroinflammation has attracted increasing attention due to its potential in the development of novel molecular biomarkers and targeted therapies $(46,47)$. In has been shown that both innate and acquired immunity play important roles in neuroinflammation in the progression of $\mathrm{PD}(48,49)$. Previous studies have shown that acquired immunity, especially $\mathrm{T}$ cell immunity, plays a key role in the immune system dysfunction of PD (50-52), but the function of B cells in PD is still not fully understood (17).

In this study, we comprehensively characterized the B cell population in patients with PD. Although some studies have reported that the level of total $\mathrm{B}$ cells remains unchanged or even decreased in patients with PD (53-55), our study shows that the B cell subpopulation structure has changed significantly. We observed significantly decreased naïve B cells and significantly increased unswitched memory B cells (especially USM2 subset) in PD patients compared to healthy controls. Notably, the two unswitched memory B cell clusters, USM1 and USM2, both harbored IGHD and highly expressed the memory marker CD27, but had distinct gene expression patterns (Figure S2A). USM2 overexpressed several B cell activation-related genes, such as FCRL5, EGR1 and CD86. FCRL5+ memory B cells are optimally responsive cells (32), have more extensive proliferative history (56), and are committed to becoming plasmablasts (57). EGR1 participates in B cell maturation as a positive regulator (33). CD86 is usually upregulated after $\mathrm{B}$ cell activation, which can then be further activated and in turn activate $\mathrm{T}$ cells (34). It is reported that unswitched memory B cells show faster and stronger re-stimulation potential and are involved in early inflammatory response (58). In this study, the USM2 subset has strong activation features and high proliferative potential. We speculate that it may be an important participant in the humoral immune response of Parkinson's disease, and may eventually contribute to the production of infiltrating antibodies in the brain of PD patients. Their potential role in Parkinson's disease deserves further investigation.

We also discovered increased IgG and IgA isotypes and more frequent CSR events in PD patients compared to healthy controls. B cells have not been detected in the brains of PD patients (16), but IgG deposition had been observed around dopaminergic neurons and Lewy bodies (19). Increased circulating IgG and IgA isotypes may contribute to the IgG detected in the brain of PD patients. Moreover, the preferential $\mathrm{V}$ and $\mathrm{J}$ gene fragments of $\mathrm{B}$ cell receptors in PD patients found in this study also further provide the evidence of convergent selection in PD. HLA-DRB5 $(59,60)$, HLA-DQA2 $(60,61)$ and HLA-DPB1 $(62)$ have been reported to be associated with PD, which were all up-regulated in memory B cells of $\mathrm{PD}$ patients compared to healthy controls in this study, suggesting the enhanced capacity of antigen presentation in $\mathrm{B}$ cells of $\mathrm{PD}$ patients.

We observed BCR-induced activation of the AP-1 transcription factor up-regulated in $\mathrm{PD}$ patients, indicating $\mathrm{B}$ cell activated in PD patients. B cells produce antibodies but can also regulate immune responses. We noted that PD patients had an increased proportion of TNF $\alpha$-producing B cells compared with healthy controls (Wilcox test, one-sided, $p=0.0147$, Figure 4F). High levels of soluble TNF $\alpha$ have been detected in the cerebrospinal fluid and postmortem brains of $\mathrm{PD}$ patients as well as in animal models of PD (63-66). And the expression level of TNF $\alpha$ in cerebrospinal fluid is a candidate risk biomarker for the detection of PD at the prodromal stage (67). Anti-TNF $\alpha$ therapy protects dopaminergic neurons (68) and reduces the incidence of Parkinson's disease (69). These findings suggest that $\mathrm{TNF} \alpha$ may be a mediator of neuronal injury and a feasible target for the treatment of PD. The relationship between the increased TNF $\alpha$ production in B cells and the progression of Parkinson's disease needs further investigation. In addition, we also found that the proportion of CXCR3-expressing B cells was significantly increased in PD patients (Wilcox test, one-sided, $p=0.0406$, Figure 4F), suggesting enhanced chemotaxis of $B$ cells in PD patients. These B cell abnormalities may contribute to the development and progression of Parkinson's disease by increasing antibody and cytokine infiltration and enhancing neuroinflammation in the central nervous system (70). Overall, our study provides a comprehensive characterization of peripheral B cells in PD patients, which provide novel insights on the humoral immune response in the pathogenesis of PD.

Although our results suggest that B cells may play a role in $\mathrm{PD}$, it is not clear whether the observed changes in acquired immunity are causal or secondary to central nervous system disorder associated with the pathogenesis of PD. Further studies, such as whether anti- $\alpha$-synuclein monoclonal antibody therapy is beneficial to patients with $\mathrm{PD}$ (71), are still needed to investigate the $\mathrm{B}$ cell immunity in the pathogenesis of $\mathrm{PD}$.

\section{MATERIALS AND METHODS}

\section{Human Participants}

In this study, we recruited eight PD patients (PD1-PD8, 50-70 years) and six healthy controls (HC1-HC6, 51-72 years). All participants had no obvious somatic disorders, such as cancer, autoimmune diseases, as well as mental and cognitive disorders. All participants were recruited from the First Affiliated Hospital of Harbin Medical University and obtained informed consent. This study was approved by the Ethics Committee in the First Affiliated Hospital of Harbin Medical University, and the approval number is No. 201985.

\section{Blood Sample Collection and Single-Cell 5' and V(D)J Sequencing}

Fresh blood samples were collected from 8 PD patients and 6 age-matched healthy controls. Then, peripheral blood mononuclear cells (PBMCs) were isolated by Percoll density 
gradient centrifugation. Next, CD19+ B cells were sorted using fluorescence-activated cell sorting (FACS). Single-cell 5' gene expression libraries and $\mathrm{V}(\mathrm{D}) \mathrm{J}$ enriched libraries were prepared according to the standard protocols provided by the $10 \mathrm{x}$ Genomics Chromium Single Cell Immune Profiling Solution. Finally, single-cell 5' gene expression and V(D)J libraries were sequenced on Illumina Noveseq 6000, providing 150 bp pairedend reads.

\section{Preprocessing of Single-Cell 5' V(D)J Sequencing Data}

Single cell $5^{\prime}$ gene expression and V(D)J data was processed by Cell Ranger pipeline (version 3.1.0) for each sample. Reference data files refdata-cellranger-GRCh38-3.0.0 and refdatacellranger-vdj-GRCh38-alts-ensembl-3.1.0 downloaded from 10x Genomics official website were used in Cell Ranger analysis pipelines for single-cell transcriptome and $\mathrm{V}(\mathrm{D}) \mathrm{J}$ data processing, separately.

\section{Cell Quality Control}

We used emptyDrops function in the $\mathrm{R}$ package dropletUtils $(72,73)$ to detect and remove empty droplets. Doublets were detected by $\mathrm{R}$ package DoubletFinder (74) with default parameters. After removing empty droplets and doublets, low-quality cells were identified based on the median absolute deviation (MAD) using isOutlier function in the $\mathrm{R}$ package scater (75). Three matrics were used to detect low-quality cells: 1) Total UMI counts per cell (library size); 2) Total detected genes per cell; 3) The proportion of mitochondrial gene counts. Please see Zhang et al. (76) for details. Finally, genes with more than 1 transcript in at least two cells were retained for further analysis.

\section{Dataset Integration and Unsupervised Clustering}

Batch effects were removed, and datasets from each sample were integrated using the standard Seurat v3 integration workflow. First, raw counts of each sample were normalized using a globalscaling normalization method NormalizeData in $\mathrm{R}$ package Seurat $(24,25)$. This method normalizes the gene expression values for each cell by the total UMI counts in the sample, then multiplies this value by a scale factor $(10,000$ by default), and logtransforms the result. Then FindVariableFeatures function in Seurat $(24,25)$ was used to identify highly variable genes to further reduce the dimensionality of the data. Next, 'anchors' between pairs of samples were identified and used to harmonize the datasets. Finally, cell cycle effects were calculated by CellCycleScoring function and regressed by ScaleData function in Seurat $(24,25)$.

\section{Cell Type Annotation}

Gene expression markers were identified by FindAllMarkers function in Seurat, which performs Wilcoxon Rank Sum test to identify differentially expressed genes for each cluster. Then, SingleR (31) was applied to enhance cell type annotation by calculating global similarity of gene expression between each cell and the reference datasets. Five bulk RNA-seq datasets of purified immune cells [The Database for Immune Cell Expression (26), Monaco Immune Cell Data (27), Human Primary Cell Atlas (28), BLUEPRINT database (29) and Novershtern Hematopoietic Data (30)] were selected as reference datasets for expression similarity based cell annotation. Spearman correlation between single cell and bulk RNA-seq expression profiles were computed, and the cell labels were transferred from bulk RNA-seq datasets to every single cell. Each dataset was used separately to calculate the correlation. Cells types were finally annotated by manually checking the cellidentity marker expression and their global similarity with the gene expression of reference datasets.

\section{Pseudo-Time Reconstruction}

Firstly, single-cell trajectory analysis was conducted by Monocle 2 (version 2.14.0), which reconstructs trajectories based on tracking expression changes. Given that the direction of pseudo-time is arbitrary, we selected naïve B cells as the beginning of the trajectory. A process of transformation from naïve $B$ cells to unswitched and switched memory B cells followed by plasma cells is clearly observed (Figure 1D). Then, RNA velocity was calculated to further characterize cell fate decisions and lineage relationships. RNA velocity was estimated by velocyto pipelines (77). Finally, the velocities were visualized on the first two principal components calculated by monocle.

\section{Differential Gene Expression Analysis}

Differentially expressed genes (DEGs) was identified by FindMarkers function in Seurat $(24,25)$ using default parameters. Only genes that were detected in at least $10 \%$ of the cells in one of the two groups were tested. When calculating the $\log \mathrm{FC}$ value, the average expression value of each group was added by 1 (where 1 represents a pseudocount) and then divided, followed by logarithmic conversion. $P$ values were estimated using two-sided Wilcoxon test, and FDR was corrected using $\mathrm{BH}$. DEGs between PD patients and healthy controls as well as gene expression markers of each cell subtypes were combined to evaluate the function of $B$ cell subtypes in the humoral immune response of PD.

\section{Statistical Analysis}

All statistical analyses and visualization were performed using $\mathrm{R}$ the statistical programming language (version 4.0.3). Two sample tests were performed using two-sided Wilcoxon rank sum test. If multiple tests were performed for a single analysis, we used $\mathrm{BH}$ procedure to correct for FDR.

\section{DATA AVAILABILITY STATEMENT}

Single-cell RNA and BCR sequencing data are available at GEO (Gene Expression Omnibus) under accession number GSE194245. Custom scripts used to analyze data in this article are available upon request to the Lead Contact. 


\section{ETHICS STATEMENT}

The studies involving human participants were reviewed and approved by the Ethics Committee in the First Affiliated Hospital of Harbin Medical University (Approval number: No. 201985). The patients/participants provided their written informed consent to participate in this study.

\section{AUTHOR CONTRIBUTIONS}

QJ, LY, and HN conceived the project. SY collected the blood samples. PW contributed to data analysis. ML, WZ, XJ, ZX, YL, $\mathrm{CX}, \mathrm{RC}, \mathrm{YH}$, and XL provided the technical support. PW wrote the manuscript. QJ reviewed and edited the manuscript. All authors contributed to manuscript revision, read, and approved the submitted version.

\section{FUNDING}

This work was funded by the National Natural Science Foundation of China (Nos. 62032007 to QJ, and Nos. 62072143 to LY).

\section{REFERENCES}

1. Armstrong MJ, Okun MS. Diagnosis and Treatment of Parkinson Disease: A Review. Jama (2020) 323(6):548-60. doi: 10.1001/jama.2019.22360

2. Reeve A, Simcox E, Turnbull D. Ageing and Parkinson's Disease: Why is Advancing Age the Biggest Risk Factor? Ageing Res Rev (2014) 14(100):19-30. doi: 10.1016/j.arr.2014.01.004

3. de Lau LM, Breteler MM. Epidemiology of Parkinson's Disease. Lancet Neurol (2006) 5(6):525-35. doi: 10.1016/s1474-4422(06)70471-9

4. Schrag A, Schott JM. Epidemiological, Clinical, and Genetic Characteristics of Early-Onset Parkinsonism. Lancet Neurol (2006) 5(4):355-63. doi: 10.1016/ s1474-4422(06)70411-2

5. Wickremaratchi MM, Ben-Shlomo Y, Morris HR. The Effect of Onset Age on the Clinical Features of Parkinson's Disease. Eur J Neurol (2009) 16(4):450-6. doi: 10.1111/j.1468-1331.2008.02514.x

6. P. s. D. Foundation: Statistics (2020). Available at: https://www.parkinson.org/ Understanding-Parkinsons/Statistics.

7. Lawson RA, Yarnall AJ, Duncan GW, Breen DP, Khoo TK, Williams-Gray $\mathrm{CH}$, et al. Cognitive Decline and Quality of Life in Incident Parkinson's Disease: The Role of Attention. Parkinsonism Relat Disord (2016) 27:47-53. doi: 10.1016/j.parkreldis.2016.04.009

8. Wong YC, Krainc D. $\alpha$-Synuclein Toxicity in Neurodegeneration: Mechanism and Therapeutic Strategies. Nat Med (2017) 23(2):1-13. doi: $10.1038 / \mathrm{nm} .4269$

9. Tan EK, Chao YX. Parkinson Disease and the Immune System - Associations, Mechanisms and Therapeutics. Nat Rev Neurol (2020) 16(6):303-18. doi: 10.1038/s41582-020-0344-4

10. Hamza TH, Zabetian CP, Tenesa A, Laederach A, Montimurro J, Yearout D, et al. Common Genetic Variation in the HLA Region is Associated With LateOnset Sporadic Parkinson's Disease. Nat Genet (2010) 42(9):781-5. doi: 10.1038/ng.642

11. Hollenbach JA, Norman PJ, Creary LE, Damotte V, Montero-Martin G, Caillier S, et al. A Specific Amino Acid Motif of HLA-DRB1 Mediates Risk and Interacts With Smoking History in Parkinson's Disease. Proc Natl Acad Sci USA (2019) 116(15):7419-24. doi: 10.1073/pnas.1821778116

\section{ACKNOWLEDGMENTS}

We thank all the participants and their families for supporting this study. We thank First Affiliated Hospital of Harbin Medical University in China for providing samples and their support with FACS sorting experiments.

\section{SUPPLEMENTARY MATERIAL}

The Supplementary Material for this article can be found online at: https://www.frontiersin.org/articles/10.3389/fimmu.2022. 814239/full\#supplementary-material

Supplementary Figure 1 | Heatmap showing the percentage of cells in each cluster annotated by three purified bulk RNA-seq datasets.

Supplementary Figure 2 | Volcano plot showing differentially expressed genes (DEGs) between unswitched memory B cells USM1 and USM2 (A), as well as between switched memory B cells SM1 and SM2 (B). The x-axis represents the fold change between groups, and the $\mathrm{y}$-axis represents $P$ values.

Supplementary Table 1 | Summary of single-cell RNA and BCR sequencing data.

Supplementary Table 2 | Differentially expressed genes and functionally enriched GO terms and KEGG pathways between PD patients and healthy controls in each B cell cluster.

12. Sulzer D, Alcalay RN, Garretti F, Cote L, Kanter E, Agin-Liebes J, et al. T Cells From Patients With Parkinson's Disease Recognize $\alpha$-Synuclein Peptides. Nature (2017) 546(7660):656-61. doi: 10.1038/nature22815

13. Lindestam Arlehamn CS, Dhanwani R, Pham J, Kuan R, Frazier A, Rezende Dutra J, et al. $\alpha$-Synuclein-Specific T Cell Reactivity Is Associated With Preclinical and Early Parkinson's Disease. Nat Commun (2020) 11(1):1875. doi: 10.1038/s41467-020-15626-w

14. Schröder JB, Pawlowski M, Meyer Zu Hörste G, Gross CC, Wiendl H, Meuth SG, et al. Immune Cell Activation in the Cerebrospinal Fluid of Patients With Parkinson's Disease. Front Neurol (2018) 9:1081. doi: 10.3389/fneur. 2018.01081

15. Wang P, Yao L, Luo M, Zhou W, Jin X, Xu Z, et al. Single-Cell Transcriptome and TCR Profiling Reveal Activated and Expanded T Cell Populations in Parkinson's Disease. Cell Discov (2021) 7(1):52. doi: 10.1038/s41421-02100280-3

16. Brochard V, Combadière B, Prigent A, Laouar Y, Perrin A, Beray-Berthat V, et al. Infiltration of CD4+ Lymphocytes Into the Brain Contributes to Neurodegeneration in a Mouse Model of Parkinson Disease. J Clin Invest (2009) 119(1):182-92. doi: 10.1172/jci36470

17. Sabatino JJJr., Pröbstel AK, Zamvil SS. B Cells in Autoimmune and Neurodegenerative Central Nervous System Diseases. Nat Rev Neurosci (2019) 20(12):728-45. doi: 10.1038/s41583-019-0233-2

18. Lira A, Kulczycki J, Slack R, Anisman H, Park DS. Involvement of the Fc Gamma Receptor in a Chronic N-Methyl-4-Phenyl-1,2,3,6Tetrahydropyridine Mouse Model of Dopaminergic Loss. J Biol Chem (2011) 286(33):28783-93. doi: 10.1074/jbc.M111.244830

19. Orr CF, Rowe DB, Mizuno Y, Mori H, Halliday GM. A Possible Role for Humoral Immunity in the Pathogenesis of Parkinson's Disease. Brain (2005) 128(Pt 11):2665-74. doi: 10.1093/brain/awh625

20. Akhtar RS, Licata JP, Luk KC, Shaw LM, Trojanowski JQ, Lee VM. Measurements of Auto-Antibodies to $\alpha$-Synuclein in the Serum and Cerebral Spinal Fluids of Patients With Parkinson's Disease. J Neurochem (2018) 145(6):489-503. doi: 10.1111/jnc.14330

21. Horvath I, Iashchishyn IA, Forsgren L, Morozova-Roche LA. Immunochemical Detection of $\alpha$-Synuclein Autoantibodies in Parkinson's 
Disease: Correlation Between Plasma and Cerebrospinal Fluid Levels. ACS Chem Neurosci (2017) 8(6):1170-6. doi: 10.1021/acschemneuro.7b00063

22. Benner EJ, Banerjee R, Reynolds AD, Sherman S, Pisarev VM, Tsiperson V, et al. Nitrated $\alpha$-Synuclein Immunity Accelerates Degeneration of Nigral Dopaminergic Neurons. PloS One (2008) 3(1):e1376. doi: 10.1371/ journal.pone. 0001376

23. Chen S, Le WD, Xie WJ, Alexianu ME, Engelhardt JI, Siklós L, et al. Experimental Destruction of Substantia Nigra Initiated by Parkinson Disease Immunoglobulins. Arch Neurol (1998) 55(8):1075-80. doi: 10.1001/ archneur.55.8.1075

24. Butler A, Hoffman P, Smibert P, Papalexi E, Satija R. Integrating Single-Cell Transcriptomic Data Across Different Conditions, Technologies, and Species. Nat Biotechnol (2018) 36(5):411-20. doi: 10.1038/nbt.4096

25. Stuart T, Butler A, Hoffman P, Hafemeister C, Papalexi E, Mauck WM, et al. Comprehensive Integration of Single-Cell Data. Cell (2019) 177(7):1888902.e21. doi: 10.1016/j.cell.2019.05.031

26. Schmiedel BJ, Singh D, Madrigal A, Valdovino-Gonzalez AG, White BM, Zapardiel-Gonzalo J, et al. Impact of Genetic Polymorphisms on Human Immune Cell Gene Expression. Cell (2018) 175(6):1701-15.e16. doi: 10.1016/ j.cell.2018.10.022

27. Monaco G, Lee B, Xu W, Mustafah S, Hwang YY, Carré C, et al. RNA-Seq Signatures Normalized by mRNA Abundance Allow Absolute Deconvolution of Human Immune Cell Types. Cell Rep (2019) 26(6):1627-40.e7. doi: 10.1016/j.celrep.2019.01.041

28. Mabbott NA, Baillie JK, Brown H, Freeman TC, Hume DA. An Expression Atlas of Human Primary Cells: Inference of Gene Function From Coexpression Networks. BMC Genomics (2013) 14:632. doi: 10.1186/14712164-14-632

29. Martens JHA, Stunnenberg HG. BLUEPRINT: Mapping Human Blood Cell Epigenomes. Haematologica (2013) 98(10):1487-9. doi: 10.3324/haematol. 2013.094243

30. Novershtern N, Subramanian A, Lawton LN, Mak RH, Haining WN, McConkey ME, et al. Densely Interconnected Transcriptional Circuits Control Cell States in Human Hematopoiesis. Cell (2011) 144(2):296-309. doi: 10.1016/j.cell.2011.01.004

31. Aran D, Looney AP, Liu L, Wu E, Fong V, Hsu A, et al. Reference-Based Analysis of Lung Single-Cell Sequencing Reveals a Transitional Profibrotic Macrophage. Nat Immunol (2019) 20(2):163-72. doi: 10.1038/s41590-0180276-y

32. Kim CC, Baccarella AM, Bayat A, Pepper M, Fontana MF. FCRL5(+) Memory B Cells Exhibit Robust Recall Responses. Cell Rep (2019) 27(5):1446-1460.e4. doi: 10.1016/j.celrep.2019.04.019

33. Oh Y-K, Jang E, Paik D-J, Youn J. Early Growth Response-1 Plays a NonRedundant Role in the Differentiation of B Cells Into Plasma Cells. Immune Network (2015) 15(3):161-6. doi: 10.4110/in.2015.15.3.161

34. Axelsson S, Magnuson A, Lange A, Alshamari A, Hörnquist EH, Hultgren O. A Combination of the Activation Marker CD86 and the Immune Checkpoint Marker B and T Lymphocyte Attenuator (BTLA) Indicates a Putative Permissive Activation State of B Cell Subtypes in Healthy Blood Donors Independent of Age and Sex. BMC Immunol (2020) 21(1):14-4. doi: 10.1186/ s12865-020-00343-2

35. Suzuki T, Kiyokawa N, Taguchi T, Sekino T, Katagiri YU, Fujimoto J. CD24 Induces Apoptosis in Human B Cells via the Glycolipid-Enriched Membrane Domains/Rafts-Mediated Signaling System. J Immunol (2001) 166(9):556777. doi: 10.4049/jimmunol.166.9.5567

36. Park CK, Shin YK, Kim TJ, Park SH, Ahn GH. High CD99 Expression in Memory T and B Cells in Reactive Lymph Nodes. J Korean Med Sci (1999) 14 (6):600-6. doi: 10.3346/jkms.1999.14.6.600

37. Qiu X, Mao Q, Tang Y, Wang L, Chawla R, Pliner HA, et al. Reversed Graph Embedding Resolves Complex Single-Cell Trajectories. Nat Methods (2017) 14(10):979-82. doi: 10.1038/nmeth.4402

38. Gitlin AD, von Boehmer L, Gazumyan A, Shulman Z, Oliveira TY, Nussenzweig MC. Independent Roles of Switching and Hypermutation in the Development and Persistence of B Lymphocyte Memory. Immunity (2016) 44(4):769-81. doi: 10.1016/j.immuni.2016.01.011

39. Gupta NT, Vander Heiden JA, Uduman M, Gadala-Maria D, Yaari G, Kleinstein SH. Change-O: A Toolkit for Analyzing Large-Scale B Cell
Immunoglobulin Repertoire Sequencing Data. Bioinformatics (2015) 31 (20):3356-8. doi: 10.1093/bioinformatics/btv359

40. Hoehn KB, Fowler A, Lunter G, Pybus OG. The Diversity and Molecular Evolution of B-Cell Receptors During Infection. Mol Biol Evol (2016) 33 (5):1147-57. doi: 10.1093/molbev/msw015

41. Seifert M, Küppers R. Human Memory B Cells. Leukemia (2016) 30(12):228392. doi: 10.1038/leu.2016.226

42. Lanzavecchia A. Antigen-Specific Interaction Between T and B Cells. Nature (1985) 314(6011):537-9. doi: 10.1038/314537a0

43. Constant S, Schweitzer N, West J, Ranney P, Bottomly K. B Lymphocytes can be Competent Antigen-Presenting Cells for Priming CD4+ T Cells to Protein Antigens in vivo. J Immunol (1995) 155(8):3734-41.

44. Weber MS, Prod'homme T, Patarroyo JC, Molnarfi N, Karnezis T, LehmannHorn K, et al. B-Cell Activation Influences T-Cell Polarization and Outcome of Anti-CD20 B-Cell Depletion in Central Nervous System Autoimmunity. Ann Neurol (2010) 68(3):369-83. doi: 10.1002/ana.22081

45. Grötsch B, Brachs S, Lang C, Luther J, Derer A, Schlötzer-Schrehardt U, et al. The AP-1 Transcription Factor Fra1 Inhibits Follicular B Cell Differentiation Into Plasma Cells. J Exp Med (2014) 211(11):2199-212. doi: 10.1084/ jem.20130795

46. Ransohoff RM. How Neuroinflammation Contributes to Neurodegeneration. Science (2016) 353(6301):777-83. doi: 10.1126/science.aag2590

47. Vogelaar CF, Mandal S, Lerch S, Birkner K, Birkenstock J, Bühler U, et al. Fast Direct Neuronal Signaling via the IL-4 Receptor as Therapeutic Target in Neuroinflammation. Sci Transl Med (2018) 10(430):eaao2304. doi: 10.1126/ scitranslmed.aao2304

48. Sun C, Zhao Z, Yu W, Mo M, Song C, Si Y, et al. Abnormal Subpopulations of Peripheral Blood Lymphocytes are Involved in Parkinson's Disease. Ann Transl Med (2019) 7(22):637. doi: 10.21037/atm.2019.10.105

49. Kwon HS, Koh SH. Neuroinflammation in Neurodegenerative Disorders: The Roles of Microglia and Astrocytes. Transl Neurodegener (2020) 9(1):42. doi: 10.1186/s40035-020-00221-2

50. Tan JSY, Chao YX, Rötzschke O, Tan EK. New Insights Into ImmuneMediated Mechanisms in Parkinson's Disease. Int J Mol Sci (2020) 21 (23):9302. doi: 10.3390/ijms21239302

51. Garretti F, Agalliu D, Lindestam Arlehamn CS, Sette A, Sulzer D. Autoimmunity in Parkinson's Disease: The Role of $\alpha$-Synuclein-Specific T Cells. Front Immunol (2019) 10:303. doi: 10.3389/fimmu.2019.00303

52. Chen Z, Chen S, Liu J. The Role of T Cells in the Pathogenesis of Parkinson's Disease. Prog Neurobiol (2018) 169:1-23. doi: 10.1016/j.pneurobio.2018.08.002

53. Bas J, Calopa M, Mestre M, Molleví DG, Cutillas B, Ambrosio S, et al. Lymphocyte Populations in Parkinson's Disease and in Rat Models of Parkinsonism. J Neuroimmunol (2001) 113(1):146-52. doi: 10.1016/s0165$5728(00) 00422-7$

54. Stevens CH, Rowe D, Morel-Kopp MC, Orr C, Russell T, Ranola M, et al. Reduced T Helper and B Lymphocytes in Parkinson's Disease. J Neuroimmunol (2012) 252 (1-2):95-9. doi: 10.1016/j.jneuroim.2012.07.015

55. Niwa F, Kuriyama N, Nakagawa M, Imanishi J. Effects of Peripheral Lymphocyte Subpopulations and the Clinical Correlation With Parkinson's Disease. Geriatr Gerontol Int (2012) 12(1):102-7. doi: 10.1111/j.1447-0594. 2011.00740.x

56. Li H, Borrego F, Nagata S, Tolnay M. Fc Receptor-like 5 Expression Distinguishes Two Distinct Subsets of Human Circulating Tissue-like Memory B Cells. J Immunol (2016) 196(10):4064-74. doi: 10.4049/ jimmunol.1501027

57. Li H, Tolnay M. FCRL4 and FCRL5 Expression Distinguishes Three Human Memory B Cell Subsets in Tonsils. J Immunol (2017) 198(1 Supplement):144.11-1.

58. Seifert M, Przekopowitz M, Taudien S, Lollies A, Ronge V, Drees B, et al. Functional Capacities of Human IgM Memory B Cells in Early Inflammatory Responses and Secondary Germinal Center Reactions. J Proc Natl Acad Sci (2015) 112(6):E546-55. doi: 10.1073/pnas.1416276112

59. Arlehamn CSL, Alcalay RN, Garretti F, Cote L, Kanter E, Agin-Liebes J, et al. Immune Response in Parkinson's Disease Driven by HLA Display of $\alpha$ Synuclein Peptides. J Immunol (2017) 198(1 Supplement):55.26-6.

60. Wissemann WT, Hill-Burns EM, Zabetian CP, Factor SA, Patsopoulos N, Hoglund B, et al. Association of Parkinson Disease With Structural and 
Regulatory Variants in the HLA Region. Am J Hum Genet (2013) 93(5):98493. doi: 10.1016/j.ajhg.2013.10.009

61. Hill-Burns EM, Factor SA, Zabetian CP, Thomson G, Payami H. Evidence for More Than One Parkinson's Disease-Associated Variant Within the HLA Region. PloS One (2011) 6(11):e27109. doi: 10.1371/journal.pone.0027109

62. Bi XA, Wu H, Xie Y, Zhang L, Luo X, Fu Y. The Exploration of Parkinson's Disease: A Multi-Modal Data Analysis of Resting Functional Magnetic Resonance Imaging and Gene Dat. Brain Imaging Behav (2020) 15(4):198696. doi: 10.1007/s11682-020-00392-6

63. Boka G, Anglade P, Wallach D, Javoy-Agid F, Agid Y, Hirsch EC. Immunocytochemical Analysis of Tumor Necrosis Factor and its Receptors in Parkinson's Disease. Neurosci Lett (1994) 172(1):151-4. doi: 10.1016/03043940(94)90684-X

64. Mogi M, Togari A, Tanaka K-i, Ogawa N, Ichinose H, Nagatsu T. Increase in Level of Tumor Necrosis Factor- $\alpha$ in 6-Hydroxydopamine-Lesioned Striatum in Rats is Suppressed by Immunosuppressant FK506. Neurosci Lett (2000) 289 (3):165-8. doi: 10.1016/S0304-3940(00)01275-1

65. Barcia C, d. Pablos V, Bautista-Hernández V, Sánchez-Bahillo Á, Bernal I, Fernández-Villalba E, et al. Increased Plasma Levels of TNF- $\alpha$ But Not of IL1 $\beta$ in MPTP-Treated Monkeys One Year After the MPTP Administration. Parkinsonism Relat Disord (2005) 11(7):435-9. doi: 10.1016/j.parkreldis.2005.05.006

66. Nagatsu T, Sawada M. Inflammatory Process in Parkinsons Disease: Role for Cytokines. Curr Pharm Design (2005) 11(8):999-1016. doi: 10.2174/1381612053381620

67. Majbour NK, Aasly JO, Hustad E, Thomas MA, Vaikath NN, Elkum N, et al. CSF Total and Oligomeric $\alpha$-Synuclein Along With TNF- $\alpha$ as Risk Biomarkers for Parkinson's Disease: A Study in LRRK2 Mutation Carriers. Trans Neurodegeneration (2020) 9(1):15. doi: 10.1186/s40035-020-00192-4

68. Harms AS, Barnum CJ, Ruhn KA, Varghese S, Treviño I, Blesch A, et al. Delayed Dominant-Negative TNF Gene Therapy Halts Progressive Loss of Nigral Dopaminergic Neurons in a Rat Model of Parkinson's Disease. Mol Ther (2011) 19(1):46-52. doi: 10.1038/mt.2010.217

69. Peter I, Dubinsky M, Bressman S, Park A, Lu C, Chen N, et al. Anti-Tumor Necrosis Factor Therapy and Incidence of Parkinson Disease Among Patients With Inflammatory Bowel Disease. JAMA Neurol (2018) 75(8):939-46. doi: 10.1001/jamaneurol.2018.0605

70. Yan Z, Yang W, Wei H, Dean MN, Standaert DG, Cutter GR, et al. Dysregulation of the Adaptive Immune System in Patients With Early-Stage Parkinson Disease. J Neurol - Neuroimmunol Neuroinflamm (2021) 8(5): e1036. doi: 10.1212/NXI.0000000000001036

71. Jankovic J, Goodman I, Safirstein B, Marmon TK, Schenk DB, Koller M, et al. Safety and Tolerability of Multiple Ascending Doses of PRX002/RG7935, an
Anti- $\alpha$-Synuclein Monoclonal Antibody, in Patients With Parkinson Disease: A Randomized Clinical Trial. JAMA Neurol (2018) 75(10):1206-14. doi: 10.1001/jamaneurol.2018.1487

72. Griffiths JA, Richard AC, Bach K, Lun ATL, Marioni JC. Detection and Removal of Barcode Swapping in Single-Cell RNA-Seq Data. Nat Commun (2018) 9(1):2667. doi: 10.1038/s41467-018-05083-x

73. Lun ATL, Riesenfeld S, Andrews T, Dao TP, Gomes T, Marioni JC, et al. EmptyDrops: Distinguishing Cells From Empty Droplets in Droplet-Based Single-Cell RNA Sequencing Data. Genome Biol (2019) 20(1):63. doi: 10.1186/ s13059-019-1662-y

74. McGinnis CS, Murrow LM, Gartner ZJ. DoubletFinder: Doublet Detection in Single-Cell RNA Sequencing Data Using Artificial Nearest Neighbors. Cell Syst (2019) 8(4):329-37.e4. doi: 10.1016/j.cels.2019.03.003

75. McCarthy DJ, Campbell KR, Lun AT, Wills QF. Scater: Pre-Processing, Quality Control, Normalization and Visualization of Single-Cell RNA-Seq Data in R. Bioinformatics (2017) 33(8):1179-86. doi: 10.1093/bioinformatics/ btw777

76. Zhang Q, He Y, Luo N, Patel SJ, Han Y, Gao R, et al. Landscape and Dynamics of Single Immune Cells in Hepatocellular Carcinoma. Cell (2019) 179(4):82945.e20. doi: 10.1016/j.cell.2019.10.003

77. La Manno G, Soldatov R, Zeisel A, Braun E, Hochgerner H, Petukhov V, et al. RNA Velocity of Single Cells. Nature (2018) 560(7719):494-8. doi: 10.1038/ s41586-018-0414-6

Conflict of Interest: The authors declare that the research was conducted in the absence of any commercial or financial relationships that could be construed as a potential conflict of interest.

Publisher's Note: All claims expressed in this article are solely those of the authors and do not necessarily represent those of their affiliated organizations, or those of the publisher, the editors and the reviewers. Any product that may be evaluated in this article, or claim that may be made by its manufacturer, is not guaranteed or endorsed by the publisher.

Copyright (อ 2022 Wang, Luo, Zhou, Jin, Xu, Yan, Li, Xu, Cheng, Huang, Lin, Yao, Nie and Jiang. This is an open-access article distributed under the terms of the Creative Commons Attribution License (CC BY). The use, distribution or reproduction in other forums is permitted, provided the original author(s) and the copyright owner(s) are credited and that the original publication in this journal is cited, in accordance with accepted academic practice. No use, distribution or reproduction is permitted which does not comply with these terms. 